\title{
Wideband Coupled Loop Antenna for Laptop PC Sensor Network Applications
}

\author{
Chien-Min Cheng, Shih-Hsien Tseng, and Wen-Shan Chen* \\ Department of Electronic Engineering, Southern Taiwan University of Science and Technology, \\ No. 1, Nan-Tai Street, Yungkang Dist., Tainan City 710, Taiwan \\ (Received September 30, 2016; accepted Junuary 6, 2017)
}

Keywords: loop antenna, laptop device, envelope correlation coefficient

In this paper, we propose a low-profile and small wideband multiple-input multiple-output (MIMO) antenna for laptop device sensor network applications, which includes long term evolution (LTE)/wireless wide area network (WWAN) bands, where operating frequencies are 698-960 and 1710-2690 MHz. The proposed antenna is composed of two subantenns: a monopole antenna and a coupled loop antenna. The proposed antenna has dimensions of $62.8 \times 11.8 \times 0.4 \mathrm{~mm}^{3}$, good antenna impedance bandwidth, nearly omnidirectional radiation patterns, antenna isolation of -15 $\mathrm{dB}$, and an ECC value of only 0.01 . Thus, it is very suitable for the sensor network applications of small LTE/WWAN band laptop devices, including modern commercial LTE (700/2300/2500), WWAN (850/900), process control system (PCS), distributed control system (DCS), and universal mobile tellecommunications system (UMTS) standards.

\section{Introduction}

Owing to the tendency to miniaturize of modern electronic devices, laptop antennas are also following the trend of small size and wideband for wider applications, and this has become a challenge to laptop antenna designers. Recently, the dominant operating bands of the most popular laptop devices have been the long term evolution (LTE)/wireless wide area network (WWAN), which operates for $2 \mathrm{G}, 3 \mathrm{G}$, and $4 \mathrm{G}$ applications. ${ }^{(1-3)}$ The operating bands of LTE include 698-960, 1710-2690, and 3400-3800 MHz. For the GSM850/900, distributed control system (DCS), process control system (PCS), and universal mobile tellecommunications system (UMTS) standards, the operating bands are 824-960 and 1710-2170 MHz. ${ }^{(3-6)}$ Hence, most laptop devices include two main wide operating bands of 698-960 and 1710-2690 MHz. ${ }^{(7,8)}$ The main design concepts of modern laptop devices are the planar inverted-F antenna (PIFA) and the coupling loop, owing to their simple structures, which reveal better impedance bandwidths as well as antenna properties. Furthermore, compared to normal antennas (single input and single output), the multiple-input multiple-output (MIMO) antennas enhance data transmission capacities and rates owing to their multi-inputs and multi-outputs; therefore, this has recently become the most widely used communication technology.

In this paper, using the MIMO antenna design technology, we propose two coupling loop subantennas (Antenna_1 and Antenna_2) to resonate lower and upper bands (698-960 and 1710 
$-2690 \mathrm{MHz}$ ), which thus can cover the operating bands of LTE/WWAN for laptop devices. ${ }^{(9-11)}$ Because impedance bandwidth, isolation, and envelope correlation coefficient (ECC) are important and practical design parameters for laptop devices, we present and investigate these parameters in this study. Finally, antenna peak gains and efficiencies are also presented and analyzed.

\section{Antenna Design}

The proposed antenna was designed on FR4 substrates (thickness $=0.4 \mathrm{~mm}, \varepsilon_{r}=4.4$ ) using a $50-\Omega$ coaxial line to feed to a metal ground $\left(166 \times 120 \mathrm{~mm}^{2}\right)$. Except for the main antenna pattern, additional metal (thickness $=3 \mathrm{~mm}$ ) was added to form a three-dimensional structure. Detailed design patterns and parameters are shown and listed in Figs. 1 and 2 and Table 1. The proposed antenna can be divided into two subantennas with the same dimensions and symmetric structure: Antenna_1 (monopole antenna) and Antenna_2 (loop antenna). In addition, Antenna_1 is the

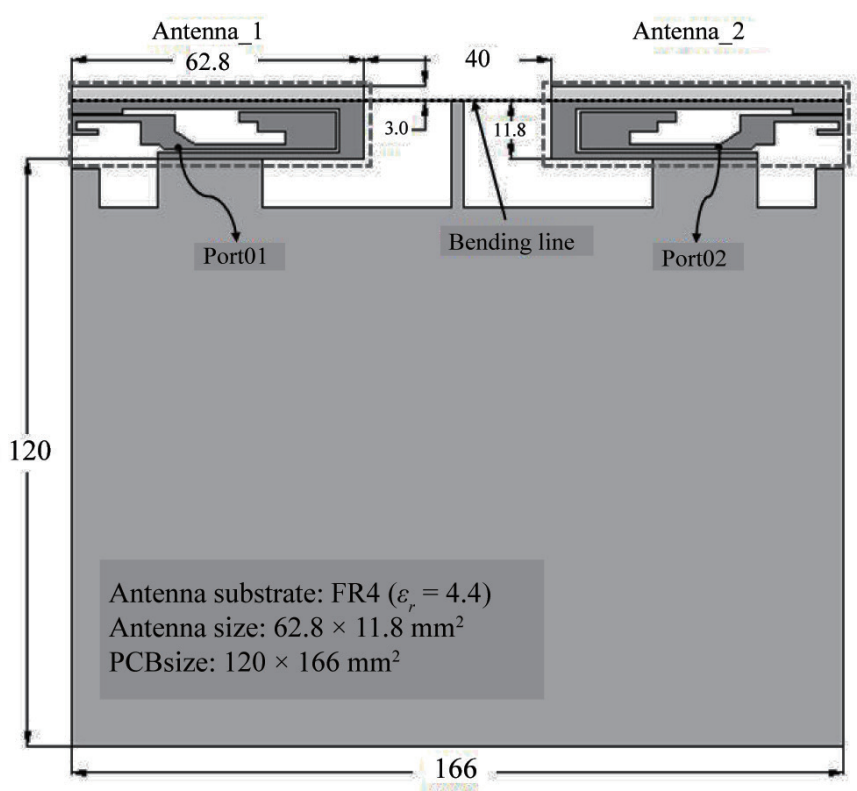

Fig. 1. Geometry of the proposed laptop antenna.

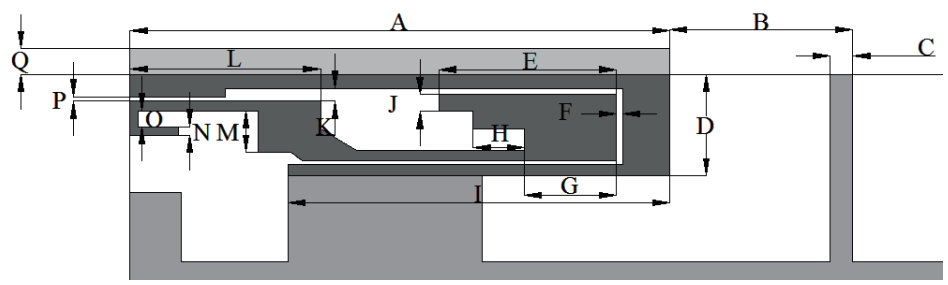

Fig. 2. Detailed structure of Antenna_1.

Table 1

Design parameters of Antenna_1 (unit: $\mathrm{mm}$ ).

\begin{tabular}{ccccccccccccccccc}
\hline $\mathrm{A}$ & $\mathrm{B}$ & $\mathrm{C}$ & $\mathrm{D}$ & $\mathrm{E}$ & $\mathrm{F}$ & $\mathrm{G}$ & $\mathrm{H}$ & $\mathrm{I}$ & $\mathrm{J}$ & $\mathrm{K}$ & $\mathrm{L}$ & $\mathrm{M}$ & $\mathrm{N}$ & $\mathrm{O}$ & $\mathrm{P}$ & $\mathrm{Q}$ \\
\hline 62.8 & 21.3 & 2.6 & 11.8 & 20.7 & 0.7 & 10.7 & 6 & 44.4 & 2 & 1.5 & 22.2 & 4.8 & 1 & 1.85 & 0.5 & 3 \\
\hline
\end{tabular}


main radiation monopole antenna composed of two inverted-L structures, which resonate and cover the upper band of 1710-2690 MHz. However, Antenna_2 is a loop antenna excited by the main radiation pattern and mutual coupling with another inverted-L antenna to the ground plane for better impedance matching and enabling it to resonate and cover the lower band of 698-960 MHz. The simulation results were obtained using the HFSS simulator, the measured results of the proposed antenna were obtained from the network analyzer, and far-field measurements including the efficiencies and peak gains were completed using the SATIMO system in a chamber.

\section{Results and Discussion}

The simulated and measured $S_{11}$ (for Antenna_1) and $S_{22}$ (for Antenna_2) are shown in Fig. 3; the $-6 \mathrm{~dB}$ dashed line for laptop antenna design is also presented. It can be seen that the proposed wideband antenna can cover both lower $(698-960 \mathrm{MHz})$ and upper (1710-2690 MHz) bands of LTE/WWAN. We also found that the simulated and measured return losses reveal a slight mismatch for both lower and upper bands because of the existence of parasitic capacitance, which causes shifts in these two operating bands. As the distance between Antenna_1 and Antenna_2 is $4 \mathrm{~cm}$, the isolation is as shown in Fig. 4. It can be observed that for both lower and upper bands, owing to the back-to-back structure of these two subantennas, the proposed antenna reveals very good isolation $(<-15 \mathrm{~dB})$. Hence, the proposed antenna possesses good isolation for reducing the mutual interference between these two subantennas.

From the ECC shown in Fig. 5, it can be seen that, from 698 to $2700 \mathrm{MHz}$, all the ECC values were very stable and varied only between 0.025 and 0.035 . Because of the smaller than 0.01 ECC value variations between Antenna_1 and Antenna_2, an improvement in the 4G channel capacity of the proposed antenna can be achieved easily. Figure 6 shows the $2 \mathrm{D}$ radiation patterns measured at 700,1800 , and $2600 \mathrm{MHz}$ for the $X Y$ plane (azimuthal plane), $X Z$ plane (elevation plane orthogonal to the device ground plane), and $Y Z$ plane (elevation plane parallel to the device ground plane), respectively. For a low frequency of $700 \mathrm{MHz}$, the antenna reveals a nearly omnidirectional

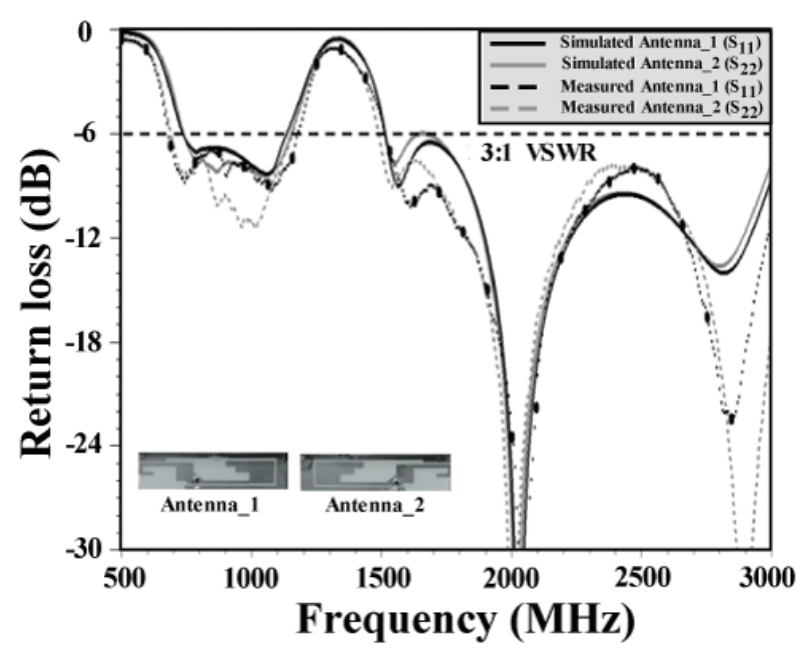

Fig. 3. Simulated and measured $S_{11}$ of Antenna_1 and $\mathrm{S}_{22}$ of Antenna_2.

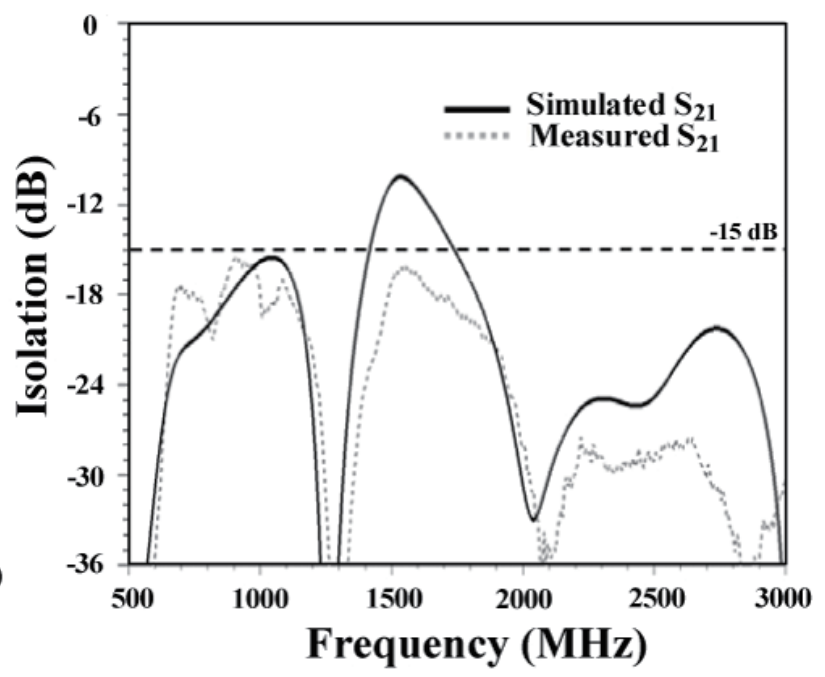

Fig. 4. Measured and simulated isolation between Antenna_1 and Antenna_2 (distance is $4 \mathrm{~cm}$ ). 


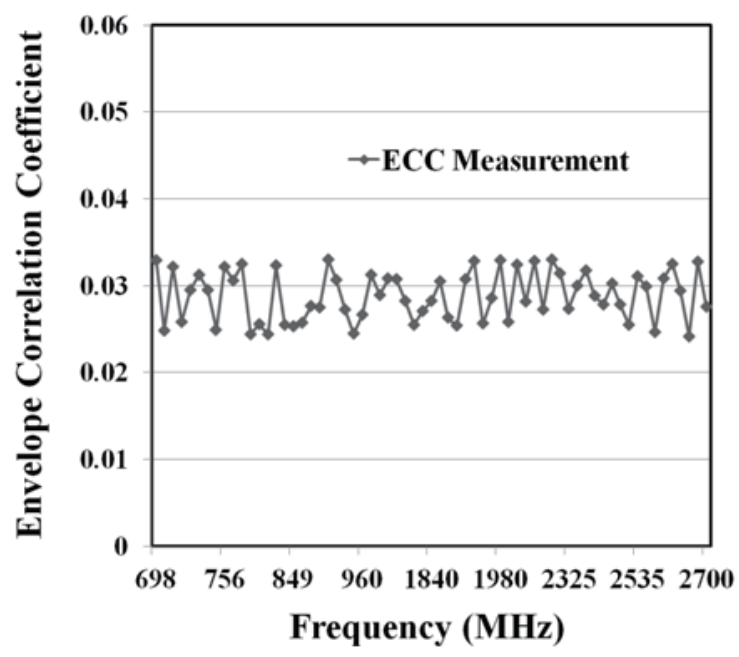

Fig. 5. Measured envelope correlation coefficient.
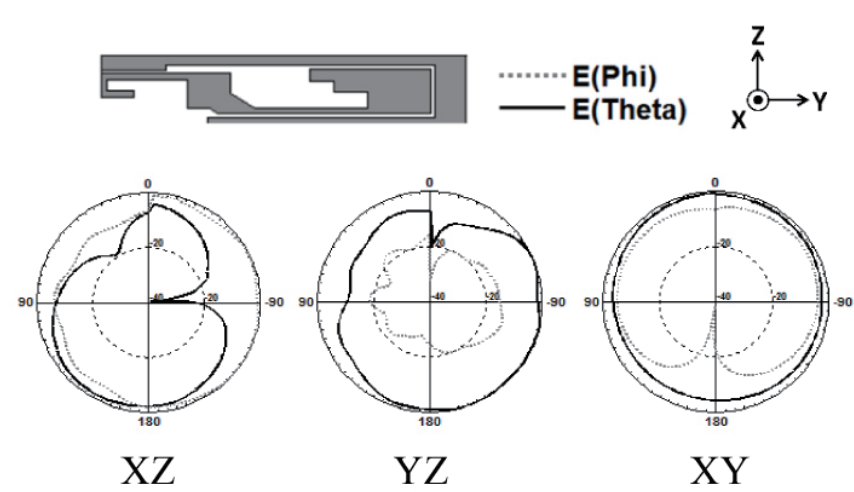

(a)

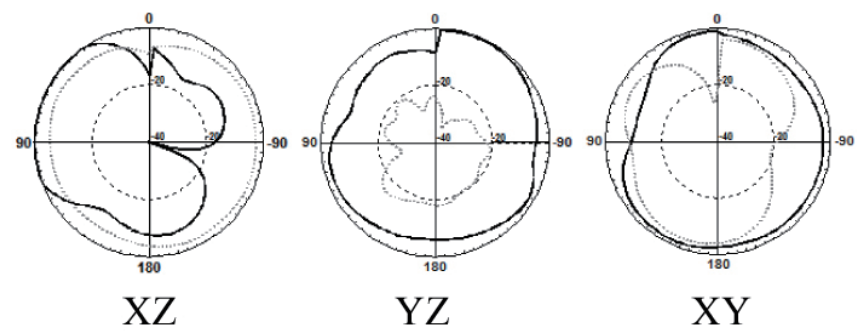

(b)

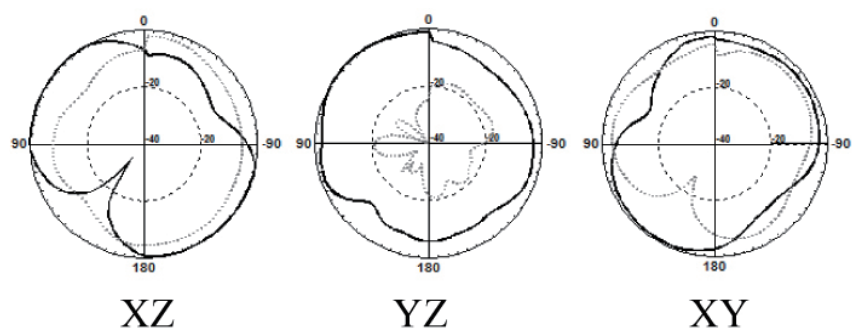

(c)

Fig. 6. Measured radiation patterns for the LTE/ WWAN coupled loop antenna. (a) 700, (b) 1800, and (c) $2600 \mathrm{MHz}$.

radiation pattern in the $X Y$ plane, which achieves good total radiated power (TRP) and total isotropic sensitivity (TIS), and better transmission and reception can be obtained for the laptop PC applications. Furthermore, for the $X Y$ plane at 1800 and $2600 \mathrm{MHz}$, variations of the radiation patterns can be found, which can be applied to the complicated propagation environment.

The simulated and measured far-field characteristics were determined using the SATIMO system in a chamber, such as antenna efficiencies (shown in Fig. 7) and peak gains (shown in Fig. 8). The lower and upper band efficiencies were 45-60 and 43-63\%, and 48-82 and 46-72\% for Antenna_1 and Antenna_2, respectively. Furthermore, the lower and upper band peak gains were 0.3-2.8 and 2.3-5.2 dBi, and 0.1-2.7 and 1.8-5.5 dBi for Antenna_1 and Antenna_2, respectively. These antenna characteristics are acceptable for modern applications, and for $4 \mathrm{G}$ fast data transmission, the antenna can increase channel capacity owing to its small variation in the ECC value. A photograph of part of the proposed antenna (Antenna_2) is presented in Fig. 9, which has miniaturized dimensions of only $62.8 \times 11.8 \mathrm{~mm}^{2}$. 


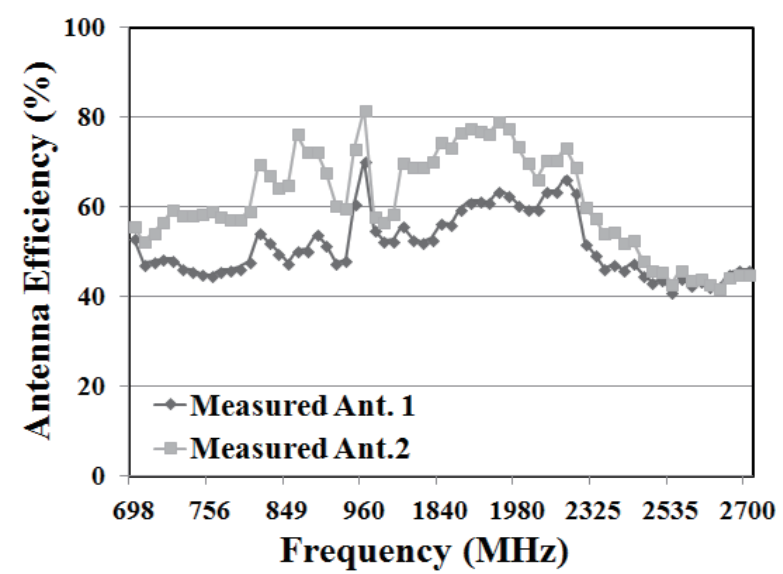

Fig. 7. Measured efficiencies of Antenna_1 and Antenna_2.

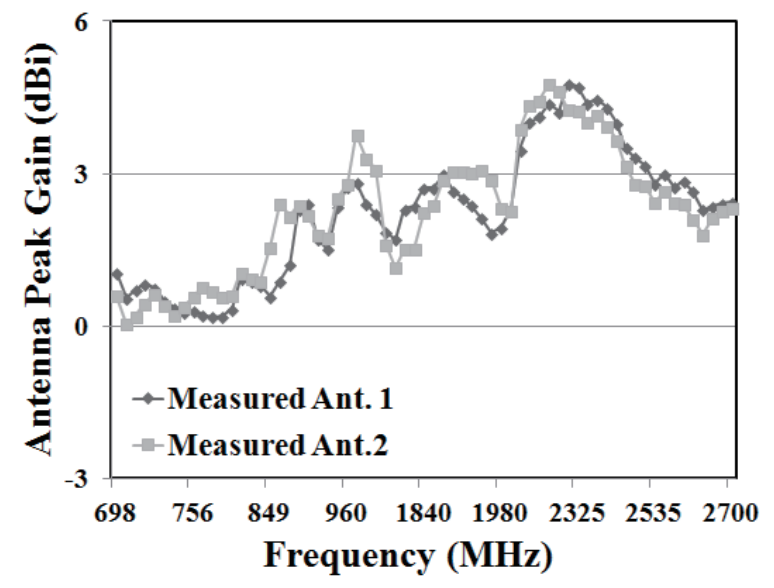

Fig. 8. Measured peak gains of Antenna_1 and Antenna_2.

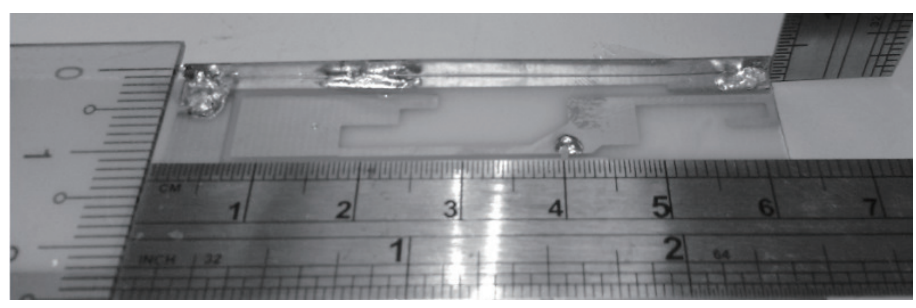

Fig. 9. Photograph of Antenna_2.

\section{Conclusions}

In this paper, a low-profile wideband coupling loop MIMO antenna for laptop device sensor network applications was presented that can cover modern LTE/WWAN bands of 698-960 MHz and 1710-2690 MHz. The proposed antenna has miniaturized dimensions of $62.8 \times 11.8 \times 0.4$ $\mathrm{mm}^{3}$, good antenna impedance bandwidth, nearly omnidirectional radiation patterns, antenna isolation of $-15 \mathrm{~dB}$, and an ECC value of only 0.01 . The optimal antenna peak gain is $4.77 \mathrm{dBi}$ and the antenna efficiency is $79 \%$; thus, the proposed antenna is very suitable for the sensor network applications of small LTE/WWAN band laptop devices.

\section{Acknowledgements}

This work was kindly supported by the Ministry of Science and Technology of the Republic of China (MOST 105-2221-E-218-034 and 105-ET-E-218-001-ET).

\section{References}

1 C. T. Lee and K. L. Wong: IEEE Trans. Antennas Propag. 58 (2015) 2479.

2 S. C. Chen and K. L. Wong: Microwave Opt. Technol. Lett. 52 (2010) 2059.

3 J. Villanen, J. Ollikainen, O. Kivekas, and P. Vainikainen: 2003 IEEE 58th Vehicular Technol. Conf. (VTC2003-Fall) p. 40. 
4 R. A. Bhatti, J. H. Choi, and S. O. Park: IEEE Antennas Wirel. Propag. Lett. 8 (2009) 129.

5 V. Plicanic, B. K. Lau, A. Derneryd, and Z. Ying: IEEE Trans. Antennas Propag. 57 (2009) 1547.

6 C. Tse and K. L. Wong: IEEE Trans. Antennas Propag. 58 (2010) 2479.

7 S. H. Chang and W. J. Liao: Int. Symp. Antennas Propag. (IEEE, 2012) p. 142.

8 A. Krewski, W. L. Schroeder, and K. Solbach: Int. Symp. Antennas Propag. (IEEE, 2012) p. 1894.

9 S. C. Chen and J. Y. Sze: Int. Symp. Antennas Propag. (IEEE, 2014) p. 425.

10 P. W. Lin, T. J. Wu, and K. L. Wong: Int. Symp. Antennas Propag. (IEEE, 2013) p. 514.

11 X. Zhu and Y. Sun: Prog. Electromagn. Res. C 42 (2013) 229.

\section{About Authors}
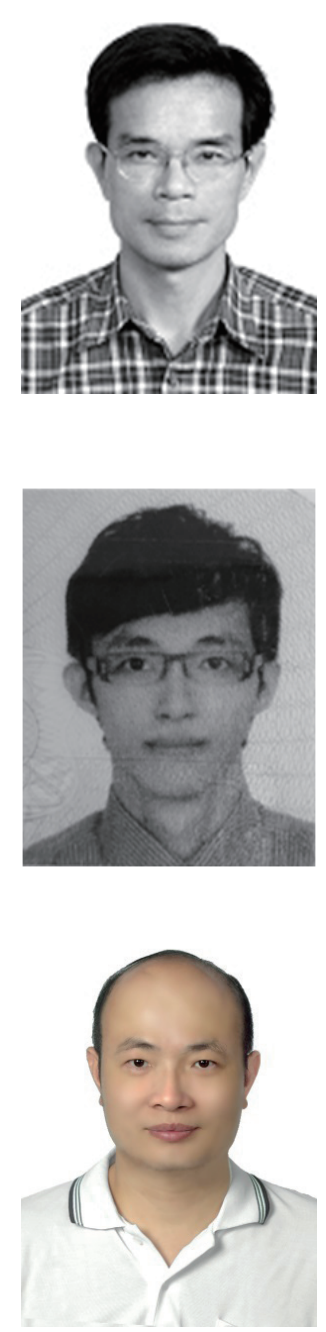

Chien-Min Cheng received his B.S., M.S., and Ph.D. degrees from the National Taiwan Ocean University, National Cheng Kung University, and National Sun Yat-Sen University, Taiwan, in 1984, 1990, and 2008, respectively. From 2009 to 2012, he was an associate professor at Southern Taiwan University of Science and Technology, Taiwan. Since 2013, he has been a professor at the same university. His research interests are in electronic ceramics, thin films, microwave device design, and piezoelectric ceramics.

Shih-Hsien Tseng received his B.S. degree from Kao Yuan University and M.S. degree from Southern Taiwan University of Science and Technology. He is currently pursuing his Ph.D. degree at Southern Taiwan University of Science and Technology. His research interests are in electronic ceramics, microwave device design, and antenna design.

Wen-Shan Chen received his B.S. degree from National Taiwan University of Science and Technology and his Ph.D. degree from National Sun Yat-Sen University, Taiwan, in 2001. He is currently a Professor of Southern Taiwan University of Science and Technology. He is an IEEE Senior Member with AP, MTT, and EMC Societies. He served as the General Secretary in 20072008, Vice Chair in 2009-1010, and Chair in 2011-2012, all for the IEEE AP-S Tainan Chapter. He is also a consultant for the IEEE AP-S Tainan Chapter. He is a member of the Institute of Antenna Engineers of Taiwan and was elected to be an AdCom member in 2008-2015. He is a member of the Chinese Microwave Association and was elected to be an AdCom member in 2015-2016. He is a member of the TIEEE, Chinese Institute of Electrical Engineering, and IEICE. His research interests include antenna design, RF, and microwave circuits. 